MODELING, IDENTIFICATION AND CONTROL, 1992, VOL. 13, NO. 3, 175-188

doi:10.4173/mic.1992.3.5

\title{
Adaptive control of ROVs with actuator dynamics and saturation
}

\author{
OLA-ERIK FJELLSTAD $\dagger$, THOR I. FOSSEN $\dagger$ \\ and OLAV EGELAND $\dagger$
}

Keywords: ROV, model reference adaptive control, actuator dynamics and saturation.

A direct model reference adaptive controller (MRAC) is derived for an underwater vehicle with significant thruster dynamics and limited thruster power. The reference model decomposition (RMD) technique is used to compensate for the thruster dynamics. A reference model adjustment (RMA) technique modifying the reference model acceleration is used to avoid thruster saturation. The design methods are simulated for the yawing motion of an underwater vehicle.

\section{Introduction}

MRAC has successfully been applied for automatic steering of ships by Amerongen (1982) and for underwater vehicles by Yuh (1990). In practical implementations actuator dynamics and saturation should be considered. Unmodeled thruster dynamics and saturation are causing severe problems in practical implementations of MRAC. This is due to the structural difference between the reference model and the process, which may lead to parameter drift and instability. This paper shows how the stability margins and the performance of the adaptive controller can be improved by modifying the reference model.

The paper is organized as follows: Section 2 describes the ROV and the actuator dynamics. Adaptive feedback linearization, the RMD and the RMA techniques are described in Section 3, while a multivariable RMD applied to ROVs is presented in Section 4. Section 5 contains a simulation study of the methods.

\section{ROV and actuator dynamics}

In this section a general model of the ROV dynamics is presented, both in a vehiclefixed and in an earth-fixed reference frame. A short description of the thruster dynamics and saturation is also given.

\subsection{ROV dynamics}

An untethered underwater vehicle can be described in 6 degrees of freedom (DOF) by the nonlinear set of equations (Fossen and Sagatun (1991))

$$
\begin{aligned}
M \ddot{q}+C(\dot{q}) \dot{q}+D(\dot{q}) \dot{q}+g(x) & =B(\dot{q}) u \\
\dot{x} & =J(x) \dot{q}
\end{aligned}
$$

Received 8 May 1992.

$\dagger$ Division of Engineering Cybernetics, The Norwegian Institute of Technology, N-7034 Trondheim, Norway.

Presented at the Second International Conference on Offshore and Polar Engineering, San Francisco, USA, 14-19 June 1992, and reprinted with permission from The International Society of Offshore and Polar Engineers. 
where $x=[x y z \phi \theta \psi]^{\mathrm{T}}$ is the position and orientation in the earth-fixed reference frame, $\dot{\boldsymbol{q}}=[\text { uvwpqr }]^{\mathrm{T}}$ is linear and angular velocities in the vehicle-fixed reference frame and $u \in \Re^{p}$ is the control vector. $M$ is a $6 \times 6$ inertia matrix, $C$ is a $6 \times 6$ matrix containing Coriolis and centripetal terms, $D$ is a $6 \times 6$ matrix containing viscous damping terms, $g$ is a vector of restoring forces, and $B$ is an $6 \times p$ input matrix given by the thruster characteristics and the thruster and the control surface configuration. $J$ is a $6 \times 6$ transformation matrix depending on the choice of coordinates.

$\boldsymbol{M}$ and $\boldsymbol{C}$ also contain the added mass derivatives. It is common to assume that $\boldsymbol{M}$ is symmetric and positive definite, i.e. $\boldsymbol{M}=\boldsymbol{M}^{\mathrm{T}}>0$. Since $\boldsymbol{D}$ contains the damping terms, it is positive definite, i.e. $D>0$.

In the rest of the paper we will assume that $\tau=B(\dot{q}) u$, hence

$$
\boldsymbol{M} \ddot{\boldsymbol{q}}+\boldsymbol{C}(\dot{\boldsymbol{q}}) \dot{\boldsymbol{q}}+\boldsymbol{D}(\dot{\boldsymbol{q}}) \dot{\boldsymbol{q}}+\boldsymbol{g}(\boldsymbol{x})=\tau
$$

where the vector $\tau$ of commanded forces and torques is the control vector. The ROV dynamics can be expressed in an earth-fixed reference frame by differentiating eqn. (2) according to

$$
\ddot{\boldsymbol{x}}=\dot{J}(x) \dot{q}+\boldsymbol{J}(x) \ddot{q} \Rightarrow \ddot{q}=J^{-1}\left(\ddot{x}-\dot{J} J^{-1} \dot{x}\right)
$$

Applied to eqn. (3) this gives

$$
M J^{-1} \ddot{x}+\left(C-M J^{-1} \dot{J}\right) J^{-1} \dot{x}+D J^{-1} \dot{x}+g=\tau
$$

Premultiplying eqn. (5) with $J^{-T}$ yields a positive definite matrix $M_{x}$ in front of $\ddot{x}$. By defining

$$
\begin{aligned}
& C(\dot{q})=C\left(J^{-1} \dot{x}\right)=C^{\prime}(\dot{x}) \\
& D(\dot{q}) D\left(J^{-1} \dot{x}\right)=D^{\prime}(\dot{x})
\end{aligned}
$$

the equations of motion can be written in an earth-fixed reference frame as

$$
M_{x}(x) \ddot{x}+C_{x}(x, \dot{x}) \dot{x}+D_{x}(x, \dot{x}) \dot{x}+g_{x}(x)=\tau_{x}
$$

where

$$
\begin{aligned}
M_{x}(x) & =J^{-\mathrm{T}} M J^{-1} \\
C_{x}(x, \dot{x}) & =J^{-\mathrm{T}}\left(C^{\prime}-M J^{-1} \dot{J}\right) J^{-1} \\
D_{x}(x, \dot{x}) & =J^{-\mathrm{T}} D^{\prime} J^{-1} \\
g_{x}(x) & =J^{-\mathrm{T}} g \\
\tau_{x} & =J^{-\mathrm{T}} \tau
\end{aligned}
$$

Here $\boldsymbol{M}_{\boldsymbol{x}}=\left(\boldsymbol{M}_{\mathrm{x}}\right)^{\mathrm{T}}>0$ and $\boldsymbol{D}_{\mathrm{x}}>0$.

\subsection{Thruster dynamics}

It is assumed that the thrusters are actuated by DC motors. An analog inner loop feedback control system of PI-type with feedback from a tachometer is used to control the propeller revolution. Hence, reduced performance due to thruster stiction and hysteresis are kept at a minimum. An alternative approach, proposed by Yoerger $e t a l$. (1990), is to use sliding control (i.e. model based control) to compensate for thruster model uncertainties. The resulting closed-loop dynamics of the motor with velocity feedback can be represented by a time constant $T_{a}$. The thruster force $\tau$ is a function of 


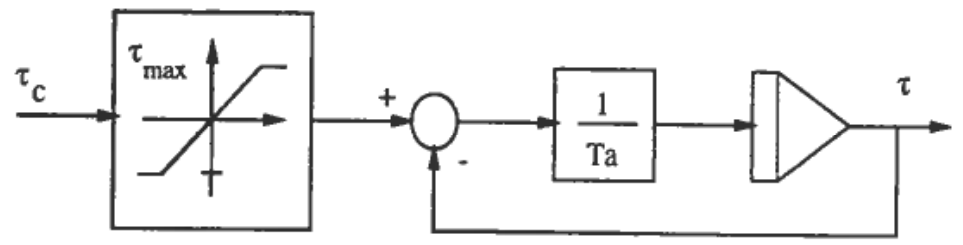

Figure 1. Thruster dynamics and saturation.

the motor velocity and has a maximum $\tau_{\max }$. A simplified block diagram of the thruster dynamics is shown in Fig. 1, where $\tau_{c}$ is the commanded input.

\section{Model reference adaptive control applied to ROVs}

A modified version of the adaptive feedback linearization scheme of Horowitz and Tomizuka (1986) will be used. The MRAC applied to ROVs is presented in Section 3.1. In Section 3.2 the RMD method of Butler $e$ t al. (1991) is outlined. This method is used in MRAC schemes to compensate for unmodeled dynamics. The last subsection shows how the reference model can be adjusted in presence of actuator saturation, which always represents a problem in physical systems. The approach here is inspired by the work of Amerongen (1982).

\subsection{Adaptive feedback linearization}

This subsection is taken from Fossen (1991), and is presented with only minor reformulation.

The adaptive feedback linearization scheme for robot manipulators is usually formulated in joint coordinates. This is due to the fact that for a large number of robot manipulators, the desired task space coordinates can be transformed to desired joint coordinates by applying the manipulator's inverse kinematics. However, in underwater vehicle control the inverse kinematics does not exist, since $\boldsymbol{\dot { q }}$ is expressed in a vehiclefixed reference frame. Nevertheless, this problem can be avoided by formulating the adaptive parameter updating law directly in the earth-fixed reference frame.

Previous work on MRAC by Horowitz and Tomizuka (1986) is based on Popov's hyperstability theory which can be used to prove global stability for the overall system. However, by applying Lyapunov-like stability theory for non-autonomous systems the derivation of the adaptive scheme is often much simpler and more intuitive. This is usually done by applying Barbălat's lemma, see e.g. Popov (1973). Hence, the laborious mathematical manipulations imposed by the Popov integral inequality and the Kalman-Yakubovitch lemma are avoided.

Again, consider the nonlinear equations of motion, eqn. (3), which for simplicity can be written as

$$
\boldsymbol{M} \ddot{q}+\boldsymbol{n}(\boldsymbol{x}, \dot{q})=\tau
$$

where

$$
n(x, \dot{q})=C(\dot{q}) \dot{q}+D(\dot{q}) \dot{q}+g(x)
$$

The control law

$$
\tau=\hat{M} a_{q}+\hat{n}(x, \dot{q})
$$


where the hat denotes the adaptive estimates and $a_{q}$ is the commanded acceleration, yields the error dynamics

$$
\boldsymbol{M}\left(\ddot{\boldsymbol{q}}-\boldsymbol{a}_{q}\right)=(\hat{\boldsymbol{M}}-\boldsymbol{M}) \boldsymbol{a}_{q}+(\hat{\boldsymbol{n}}-\boldsymbol{n})
$$

Assuming the underwater vehicle equations to be linear in their parameters, the following parametrization can be applied

$$
(\hat{M}-M) a_{q}+(\hat{n}-n)=\Phi\left(x, \dot{q}, a_{q}\right) \tilde{\theta}
$$

where $\tilde{\boldsymbol{\theta}}=\hat{\boldsymbol{\theta}}-\boldsymbol{\theta}$ is the parameter error vector, and $\boldsymbol{\Phi}$ is the regressor matrix.

The commanded acceleration $\boldsymbol{a}_{q}$ in the vehicle-fixed reference frame can be related to the earth-fixed acceleration $a_{x}$ through eqn. (4) as

$$
\boldsymbol{a}_{\boldsymbol{x}}=\dot{\boldsymbol{J}} \dot{\boldsymbol{q}}+\boldsymbol{J} \boldsymbol{a}_{\boldsymbol{q}}
$$

Applying this relation to the error dynamics gives

$$
M J^{-1}\left(\ddot{x}-a_{x}\right)=\Phi\left(x, \dot{q}, a_{q}\right) \ddot{\theta}
$$

Premultiplying with $J^{-T}$, the symmetric, positive definite matrix $\boldsymbol{M}_{x}$ from eqn. (8) is obtained, and the $x$-frame error dynamics becomes

$$
M_{x}\left(\ddot{x}-a_{x}\right)=J^{-T} \Phi\left(x, \dot{q}, a_{q}\right) \tilde{\theta}
$$

The commanded acceleration vector can be chosen by pole placement as

$$
\boldsymbol{a}_{x}=\ddot{\boldsymbol{x}}_{d}-2 \lambda \dot{\tilde{x}}-\lambda^{2} \tilde{\mathrm{x}} \quad \lambda>0
$$

Defining a measure of tracking (Slotine and $\mathrm{Li}(1987)$ )

$$
\boldsymbol{s}=\dot{\tilde{\boldsymbol{x}}}+\lambda \tilde{\boldsymbol{x}}
$$

the error dynamics can be expressed as

$$
\boldsymbol{M}_{\boldsymbol{x}}(\dot{\boldsymbol{s}}+\lambda \boldsymbol{s})=\boldsymbol{J}^{-\mathrm{T}} \boldsymbol{\Phi}\left(\boldsymbol{x}, \dot{q}, a_{q}\right) \tilde{\theta}
$$

To prove global stability a Lyapunov-like function candidate is used

$$
V(s, \tilde{\theta}, t)=\frac{1}{2}\left(s^{\mathrm{T}} M_{x} s+\tilde{\theta}^{\mathrm{T}} \Gamma \tilde{\theta}\right)
$$

Differentiating $V$ with respect to time (assuming $\dot{M}_{x}=0$ ) yields

$$
\dot{V}=s^{\mathrm{T}} M_{x} \dot{s}+\dot{\boldsymbol{\theta}}^{\mathrm{T}} \boldsymbol{\Gamma} \tilde{\boldsymbol{\theta}}
$$

where $\boldsymbol{\Gamma}$ is a diagonal positive definite weighting matrix of appropriate dimension. Substituting the error dynamics into the expression for $\dot{V}$ yields

$$
\dot{V}=-\lambda s^{\mathrm{T}} M_{x} s+\left(s^{\mathrm{T}} J^{-\mathrm{T}} \Phi+\dot{\boldsymbol{\theta}}^{\mathrm{T}} \boldsymbol{T}\right) \tilde{\theta}
$$

This suggests the parameter update law (assuming $\dot{\theta}=0$ )

$$
\hat{\theta}=-\Gamma^{-1} \Phi^{\mathrm{T}}\left(x, \dot{q}, a_{q}\right) J^{-1}(x) s
$$

which finally yields

$$
\dot{V}=-\lambda s^{\mathrm{T}} M_{x} s \leqslant 0
$$

Hence, global stability and asymptotic convergence of $\tilde{\boldsymbol{x}}$ to zero are guaranteed by applying Barbălat's Lyapunov-like lemma.

According to eqns. (16), (19) and (22) this is a series-type MRAC, i.e. the reference signal is filtered before it is fed to the controller. This is utilized in Section 3.3 where the reference model is adjusted with respect to actuator saturation. 


\subsection{Reference model decomposition}

Unmodeled dynamics in the plant affects a MRAC scheme in two ways. First, the perfect model matching conditions are violated, which means that a set of correct parameters may not exist. Second, the linear part of the scheme may no longer be strictly positive real (SPR). However, this will not necessarily cause instability.

The RMD method of Butler et al. (1991) is a technique for making MRAC schemes more robust in presence of unmodeled dynamics. The idea is to modify the reference model such that the influence of the unmodeled dynamics on the parameter adaption is reduced. Note, unmodeled does not necessarily mean completely unknown. In most cases the frequency range of the unmodeled dynamics is well known, and this can be utilized in the control design. However, it is important to keep the order of the primary controller low, and the number of updated parameters at a minimum. Violating this may lead to non-robust adaption and low performance of the controller.

Consider a linear plant with the transfer function $h(s)$, which can be separated into a nominal part $h_{n}(s)$ and an unmodelled part $h_{u}(s)$ as

$$
h(s)=h_{n}(s) h_{u}(s)
$$

$h_{u}(s)$ can always be decomposed into

$$
h_{u}=\frac{b_{1}(s)}{a(s)-b_{2}(s)}
$$

where $a(s), b_{1}(s)$ and $b_{2}(s)$ are polynomials. The decomposition is shown in Fig. 2 . Let $\overline{W_{p}}(s)$ denote the complete process transfer function

$$
\overline{W_{p}}=W_{p}+\tilde{W}_{p}
$$

and $W_{m}$ the reference model based on the nominal part $W_{p}$. Figure 3 now illustrates how the decomposition is used in a MRAC scheme.

Notice that $b_{1} /\left(a-b_{2}\right)$ represents the unmodeled dynamics in the ideal case. $a(s)$ is chosen to be of equal order as the unmodeled dynamic denominator which makes the transfer function $b_{2} / a$ realizable. When $a(s)$ is chosen, $b_{1}(s)$ and $b_{2}(s)$ are determined according to eqn. (31). Because the unmodelled dynamics is not exactly known, $a(s)$ must be tuned to give good performance in closed-loop.

One effect of the extra decomposition structure is that the linear part of the MRAC scheme will have increased gain margin. This is obtained because the real part of the transfer function is made positive in a larger frequency range. In addition, the output error is less influenced by the unmodeled dynamics, due to the modification of the model reference. This is described more closely in Butler et al. (1991).

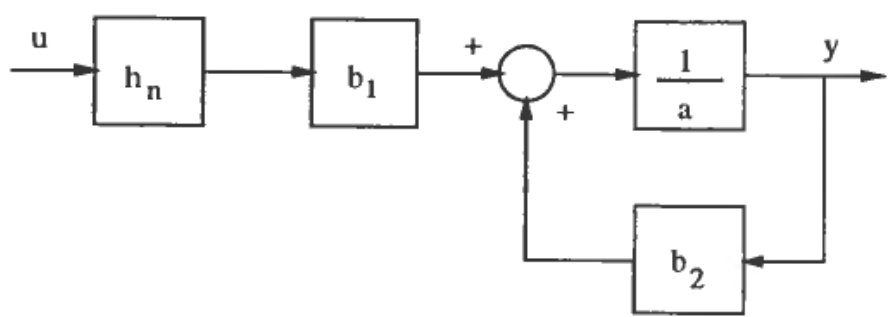

Figure 2. Decomposition defined by eqn. (31). 


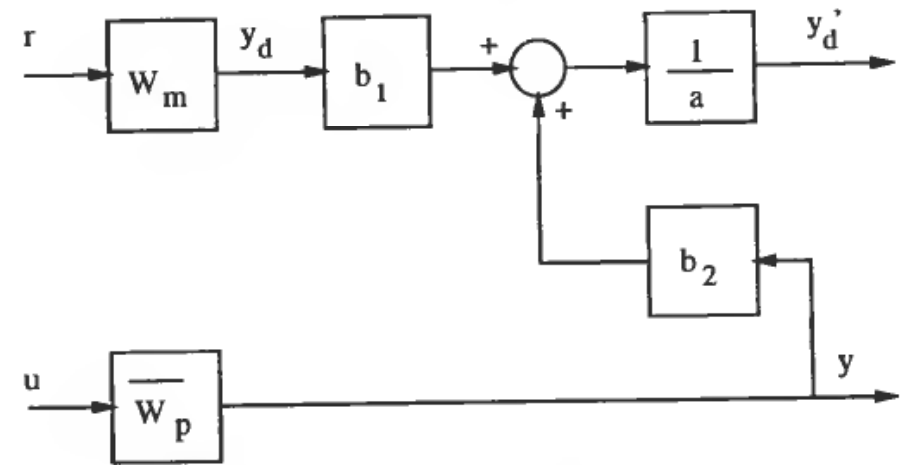

Figure 3. The decomposition used in MRAC.

\subsection{MIMO RMA}

The RMA technique can be used to compensate for actuator saturation. This is obtained by decreasing the gain in the reference model, which has the same effect as decreasing the desired acceleration, see Fig. 5. Hence, tracking of the desired states require less control action and saturation is avoided. During saturation, the RMA matrix $F_{a}$ is chosen as a diagonal matrix of positive ratios

$$
\boldsymbol{F}_{a}=\left\{f_{a, i}\right\}=\operatorname{diag}\left\{\min \left\{\frac{\tau_{\max }}{\left|\tau_{c, i}\right|}, 1\right\}\right\}
$$

Here $\tau_{\max }$ is the maximum thrust and $\tau_{c, i}$ is the commanded thrust corresponding to thruster $i$. Notice that if no saturation occurs, $f_{a, i}=1$. This is illustrated in Fig. 4.

\section{RMD applied to ROVs}

We propose to apply the RMD method in a multivariable controller for an underwater vehicle. This extension is used together with the feedback linearization scheme of Section 3.1 .

\subsection{MIMO RMD}

Defining the reference model as six second order filters, one for each state variable $x_{i}$, suggests

$$
\begin{gathered}
\ddot{x}_{d, i}+2 \zeta_{i} \omega_{0, i} \dot{x}_{d, i}+\omega_{0, i}^{2} x_{d, i}=\omega_{0, i}^{2} r_{i} \\
y_{d, i}=\dot{x}_{d, i}+\lambda_{i} x_{d, i}
\end{gathered}
$$

where all six pairs of eigenvalues can be chosen independently. Here $r_{i}$ is the reference input corresponding to state $x_{i}$. Figure 5 shows the model with the RMA factor $f_{a, i}$.

If $\lambda_{i} \leqslant \omega_{0, i}$, this yields a SPR transfer matrix $W_{m}(s)$ defined as

$$
W_{m}(s)=\operatorname{diag}\left\{\frac{\omega_{0, i}^{2}\left(s+\lambda_{i}\right)}{s^{2}+2 \zeta_{i} \omega_{0, i} s+\omega_{0, i}^{2}}\right\}
$$

According to the actuator dynamics in Section 2.2 the decomposition should reflect the following transfer matrix

$$
H_{u}(s)=\operatorname{diag}\left\{\frac{1}{T_{m} s+1}\right\}
$$




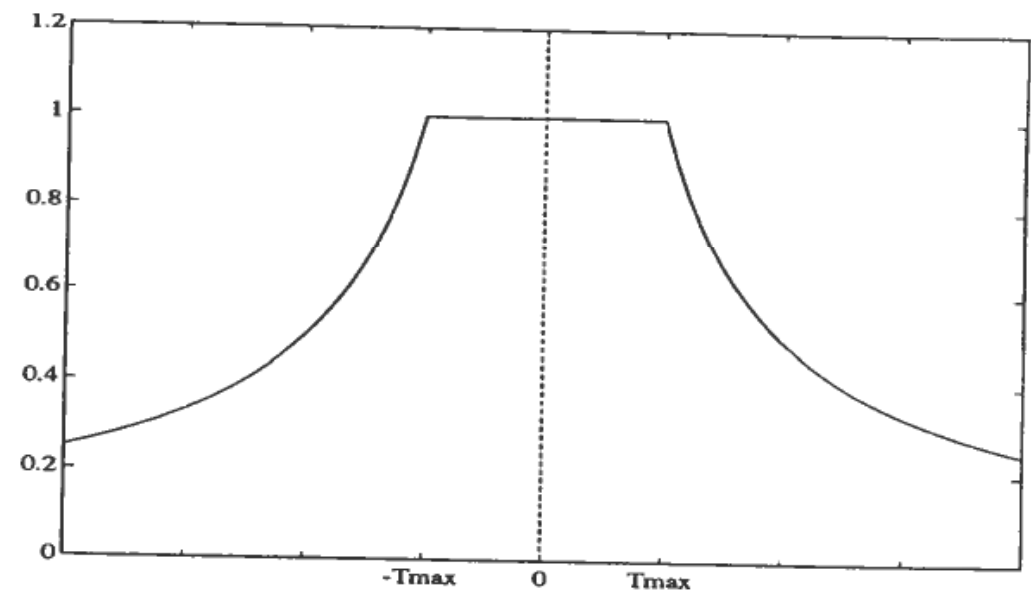

Figure 4. Adjustment factor $f_{a, i}$ plotted versus $\tau_{c, i}$.

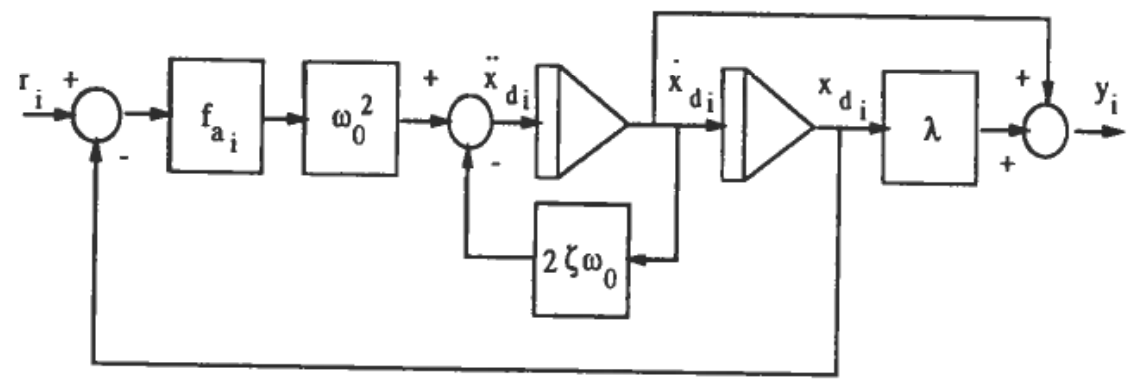

Figure 5. SISO reference model with the RMA factor $f_{a, i}$.

where $T_{m}$ is an estimate of the velocity loop time constant $T_{a}$. Defining the diagonal transfer matrices

$$
\begin{aligned}
A(s) & =\operatorname{diag}\left\{T_{n, i} s+1\right\} \\
B_{1}(s) & =I \\
B_{2}(s) & =\operatorname{diag}\left\{\left(T_{n, i}-T_{m}\right) s\right\}
\end{aligned}
$$

yields the desired decomposition

$$
\left(I-A^{-1} B_{2}\right)^{-1} A^{-1} B_{1}=H_{u}
$$

Here it is assumed that all thrusters have the same dynamics. A block diagram of the total system is shown in Fig. 6. According to the figure the reference model can be expressed as

where $\Lambda=\operatorname{diag}\left\{\lambda_{i}\right\}$ and

$$
W_{m}=(s I+\Lambda) W_{m}^{\prime}
$$

$$
W_{m}^{\prime}(s)=\operatorname{diag}\left\{\frac{\omega_{0, i}^{2}}{s^{2}+2 \zeta_{i} \omega_{0, i} s+\omega_{0, i}^{2}}\right\}
$$




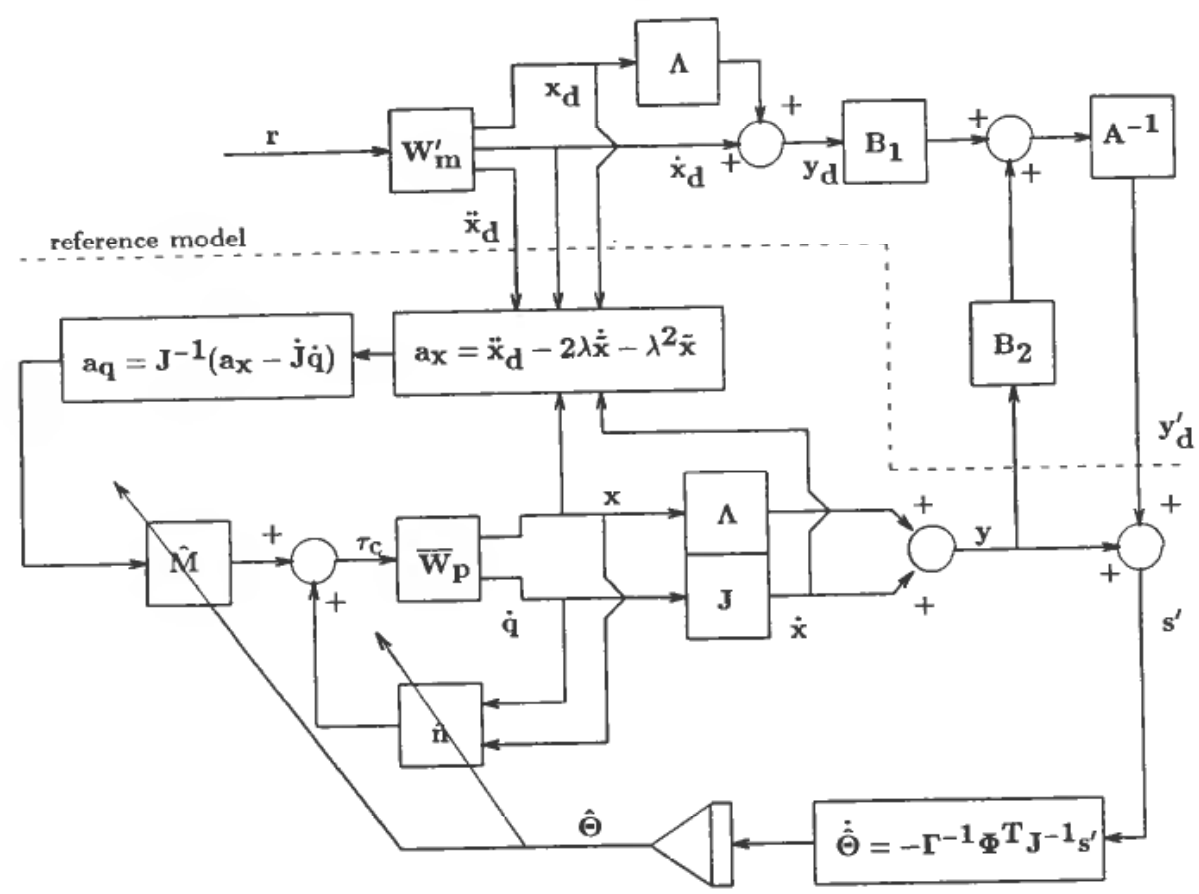

Figure 6. MRAC based on RMD.

Also note, when applying RMD the parameter update law is changed to

$$
\hat{\boldsymbol{\theta}}=-\boldsymbol{\Gamma}^{-1} \boldsymbol{\Phi}^{\mathrm{T}} \boldsymbol{J}^{-1} \boldsymbol{s}^{\prime}
$$

where

$$
\begin{aligned}
s^{\prime} & =y-y_{d}^{\prime} \\
& =y-\left(A^{-1} B_{1} y_{d}+A^{-1} B_{2} y\right) \\
& =\left(I-A^{-1} B_{2}\right) y-A^{-1} B_{1} y_{d}
\end{aligned}
$$

and $\boldsymbol{y}_{d}^{\prime}$ is the compensated model reference output.

\section{Case study: simplified model of an ROV in yaw}

The methods were examined in simulations using a simplified model of an ROV in yaw. The following experiments were run:

1. Nominal case (no thruster dynamics nor saturation).

2. Thruster dynamics, no compensation.

3. Thruster dynamics, compensation by RMD.

4. Thruster saturation, no compensation.

5. Thruster saturation, compensation by RMA.

6. Thruster dynamics and saturation, compensation by RMD and RMA.

When considering only one DOF both the Coriolis and centripetal terms vanish. Neglecting the rolling and pitching motion implies that the steering dynamics can be expressed as

$$
m \dot{\psi}+d \dot{\psi}=\tau
$$


where $\psi$ is the heading angle and

$$
y=\psi+\lambda \psi
$$

$$
\begin{gathered}
\tau=\hat{m} a_{\psi}+\hat{d} \psi=\left[a_{\psi} \psi\right]\left[\begin{array}{l}
\hat{m} \\
\hat{d}
\end{array}\right]=\boldsymbol{\phi} \hat{\boldsymbol{\theta}} \\
\hat{\boldsymbol{\theta}}=-\boldsymbol{\Gamma}^{-1} \boldsymbol{\phi}^{\mathbf{T}} \boldsymbol{s}
\end{gathered}
$$

where $s=y-y_{d}$ is the difference between the system output $y$ and the reference model output $y_{d}$. When RMD is applied, $s$ is replaced by $s^{\prime}$ in eqn. (49).

The reference model was chosen as a critically damped second order system. The parameter updating law was modified with the following normalization factor

$$
\phi \rightarrow \frac{\phi}{1+\phi^{\mathrm{T}} \phi}
$$

to improve robustness.

\subsection{The nominal case}

The following parameters were chosen as constants in all simulations:

$$
\begin{aligned}
m & =400 \mathrm{~kg} \mathrm{~m}^{2} \\
d & =100 \mathrm{~kg} \mathrm{~m}^{2} / \mathrm{s} \\
\Gamma^{-1} & =\operatorname{diag}\{1000,100\} \\
\lambda & =0.5 \\
\omega_{0} & =0.25 \mathrm{rad} / \mathrm{s} \\
\zeta & =1.0
\end{aligned}
$$

moment of inertia

dissipation

weighting matrix

closed loop bandwidth

natural frequency

relative damping

The sensor considered was the Motion Reference Unit by Seatex a.s., Pissenteret, N7005 Trondheim, Norway which contains accelerometers, inertial rotation sensors and a magnetometer. The output data rate is up to $50 \mathrm{~Hz}$, and therefore all simulations were

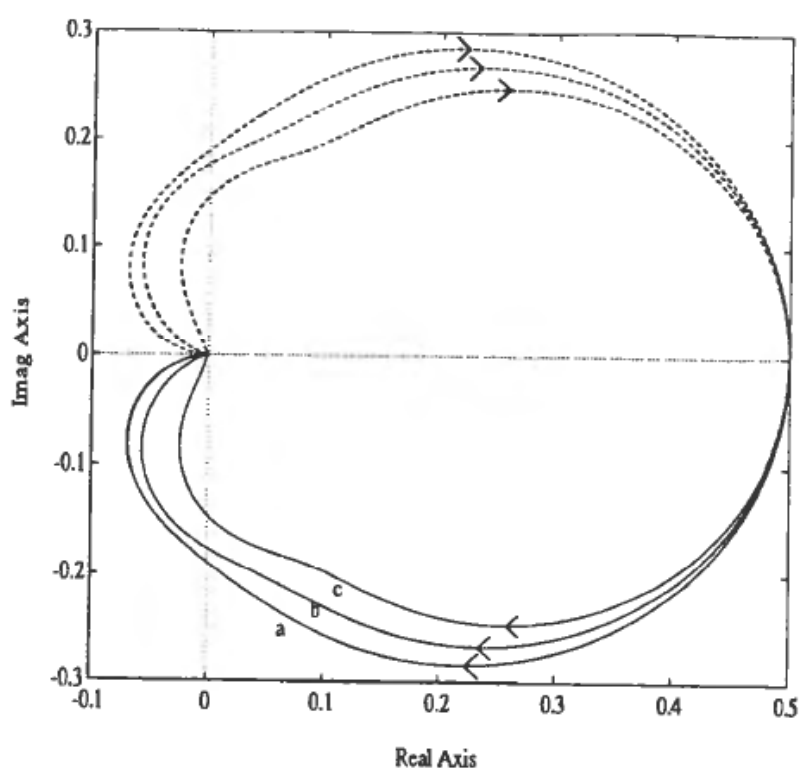

Figure 7. The output error transfer function for $(a) T_{n}=T_{m}=1,(b) T_{n}=0.5$ and (c) $T_{n}=0.05$. 

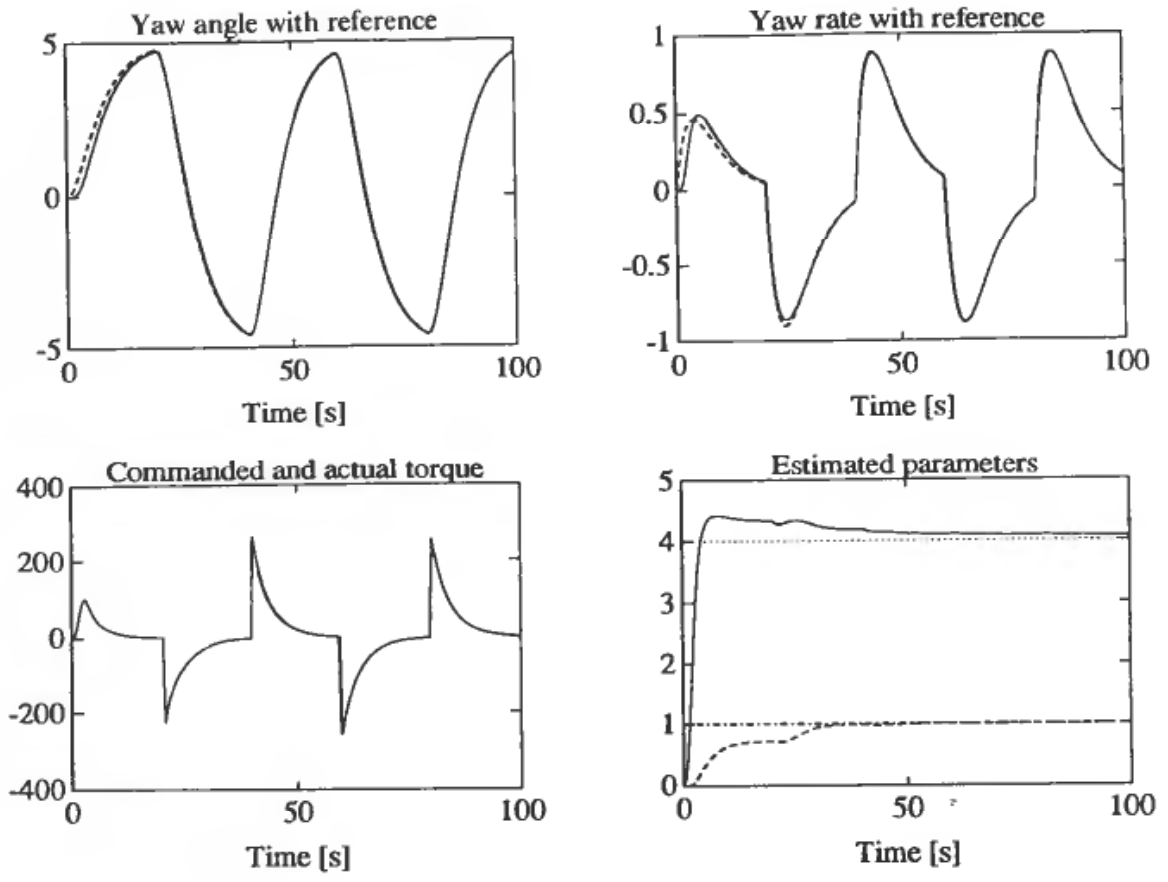

Figure 8. Case I: No thruster dynamics nor saturation.
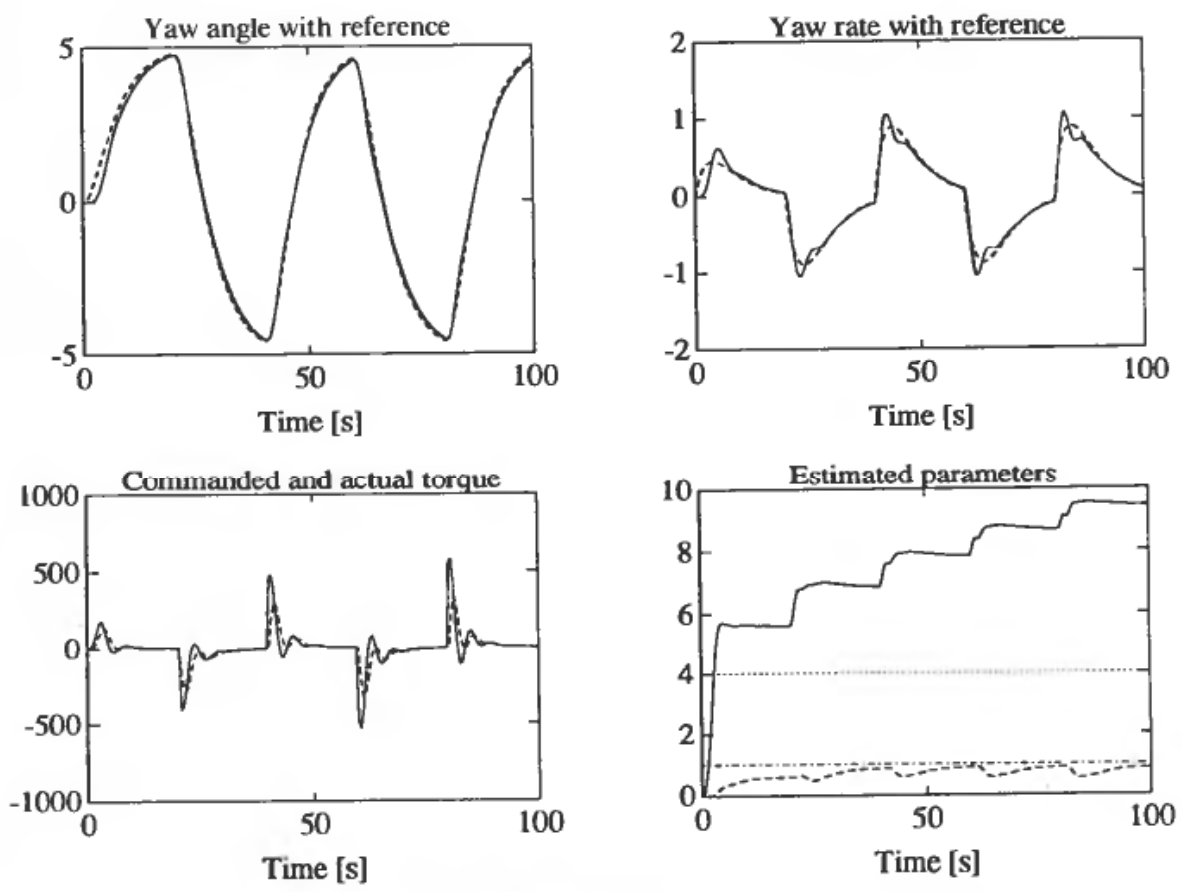

Figure 9. Case II: Thruster dynamics. No compensation. 

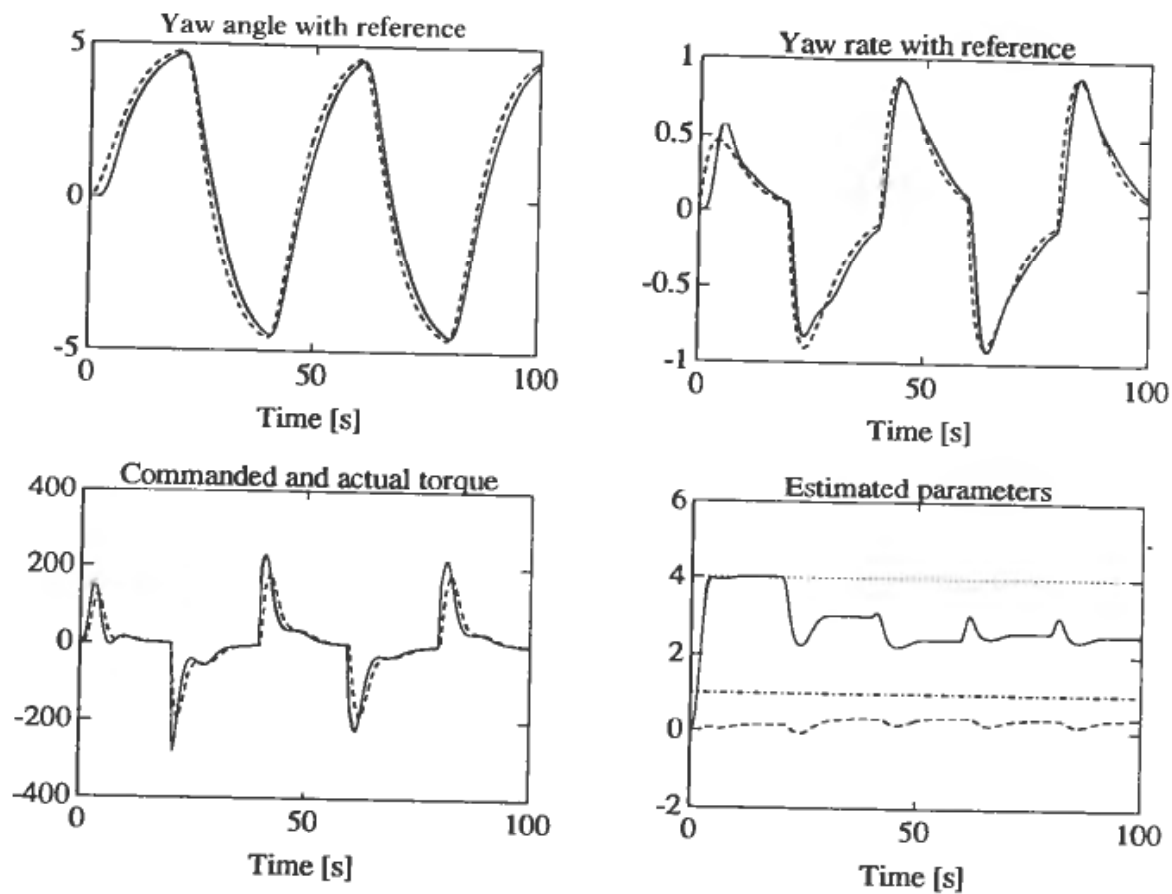

Figure 10. Case III: Thruster dynamics. Compensation by RMD.
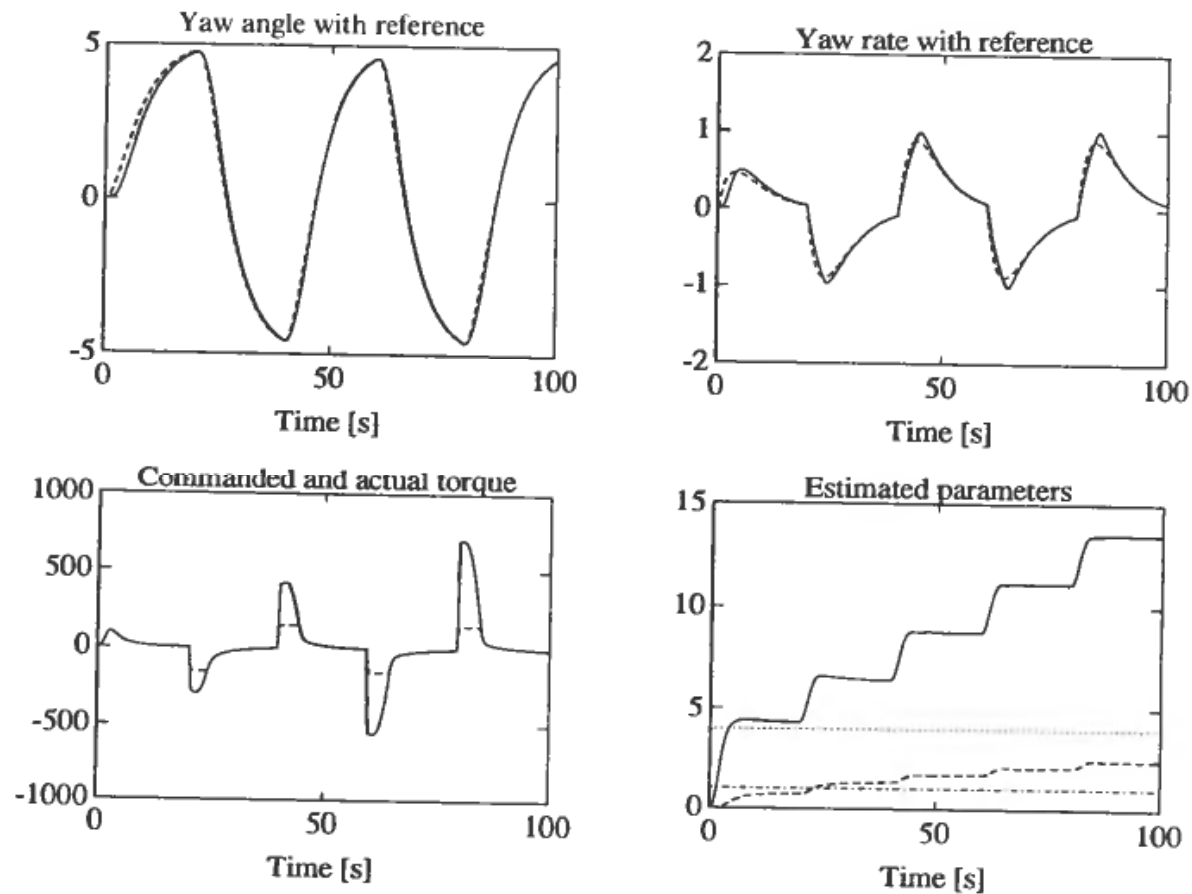

Figure 11. Case IV: Thruster saturation. No compensation. 

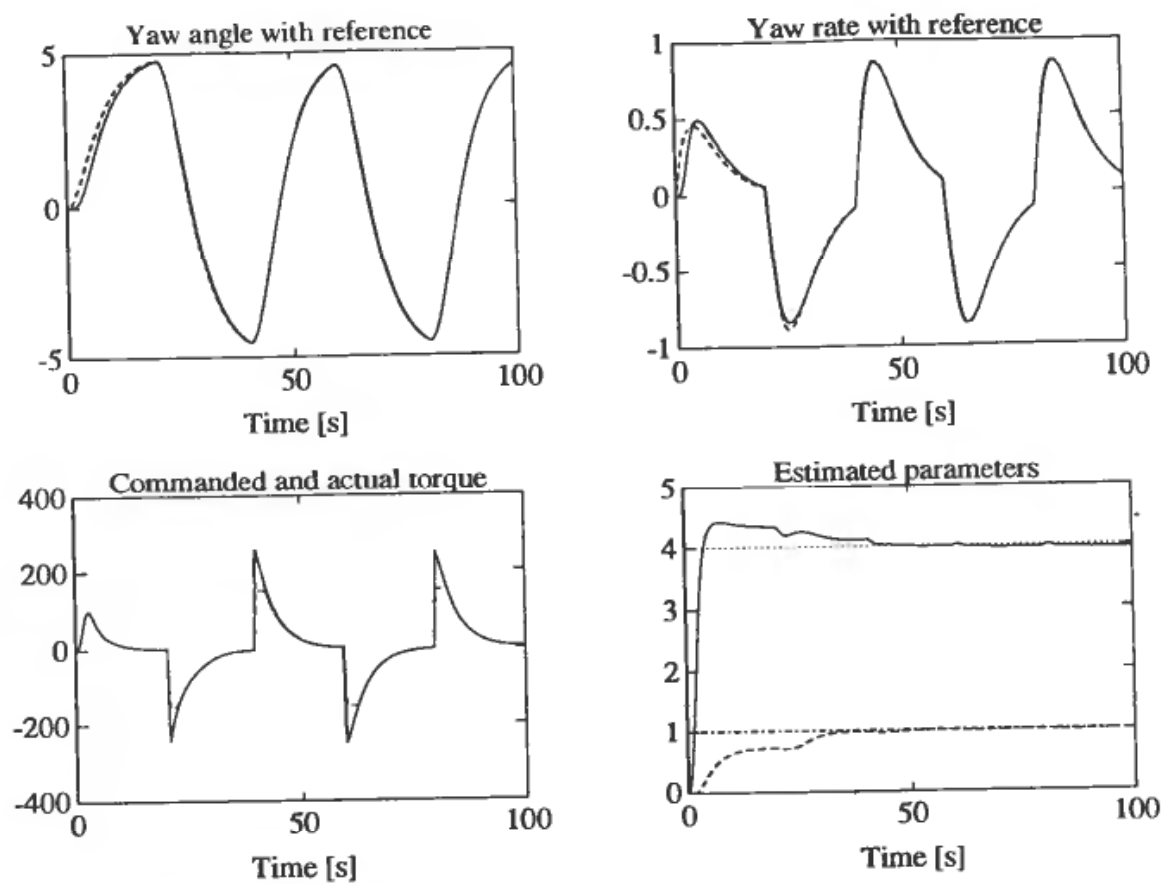

Figure 12. Case V: Thruster saturation. Compensation by RMA.
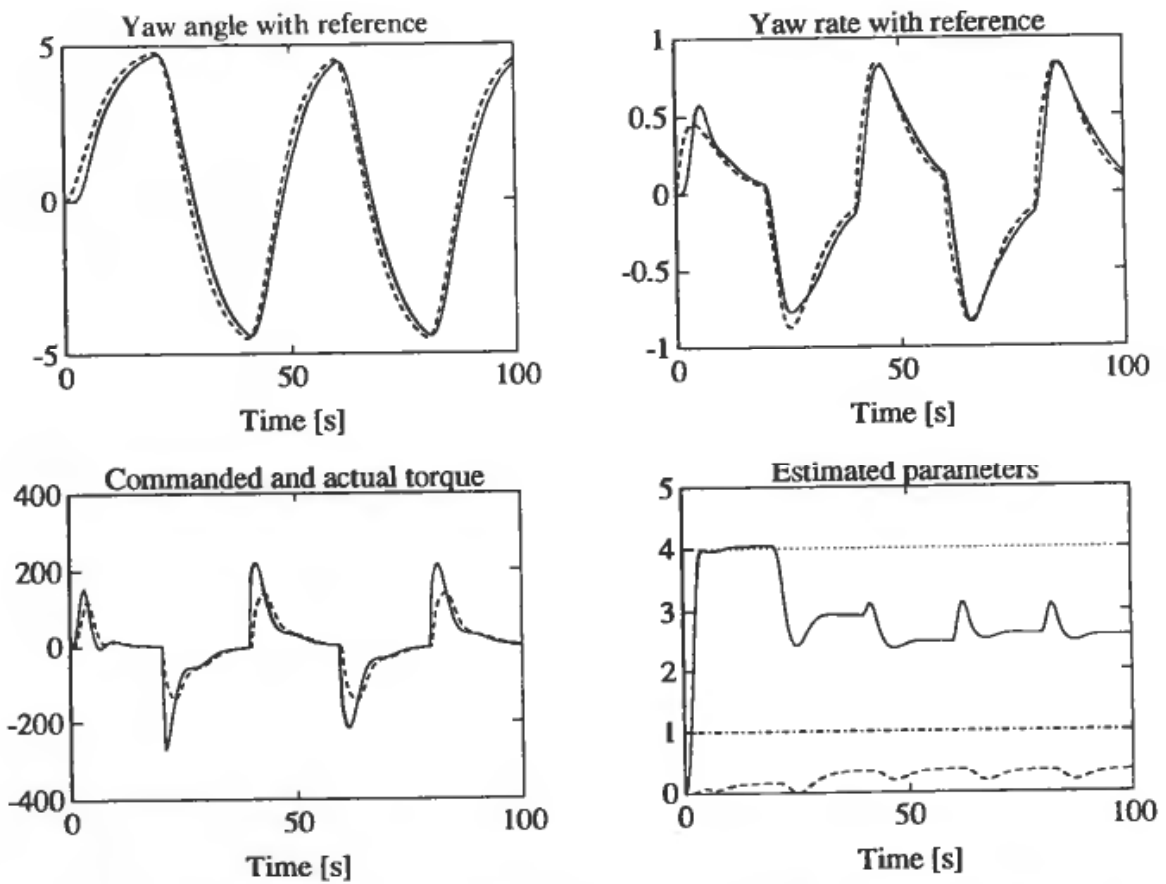

Figure 13. Case VI: Thruster dynamics and saturation. Compensation by RMD and RMA. 
run with a sampling time of $0.02 \mathrm{~s}$. In all figures the reference trajectory is dotted while the solid line represents the vehicle's motion. The commanded and actual torque are denoted with a solid and dotted line, respectively.

\section{Case I: No thruster dynamics nor saturation}

Since the perfect model matching conditions were met, and the system was persistently excited, both parameters converged to their correct values, see Fig. 8 . Notice that the parameters were scaled with a factor $1: 100$.

\subsection{Thruster dynamics}

\section{Case II: No compensation}

The thruster dynamics was modelled with a time constant $T_{a}=1[\mathrm{~s}]$. Hence, extra phase lag was introduced. In this case, the parameter estimates did not converge to their true values, see Fig. 9, and the total system might even go unstable.

\section{Case III: Compensation by RMD}

The parameter drift in Case II was removed by applying the RMD method with $T_{m}=T_{a}=1[\mathrm{~s}]$ and $T_{n}=0.05[\mathrm{~s}]$. According to Fig. 10 the estimated parameters converged, but not to their true values. Also note the reduced control action compared to Case II. Nyquist plots of the output error transfer function for this scheme is shown in Fig. 7, for various choices of $T_{n}$. The decomposition effect is illustrated by the improved phase margin for large frequencies.

\subsection{Thruster saturation}

Case IV: No compensation

The thruster was assumed to have limited thruster force, that is $\tau_{\max }=150$ [N]. This introduced parameter drift during saturation (Fig. 11), a problem similar to integral wind-up.

\section{Case V: Compensation by RMA}

The reference model was modified by applying RMA with an adjustment factor as shown in Fig. 4. This clearly reduced the occurrence of saturation. The convergence of the parameter estimates was also improved, see Fig. 12.

\subsection{Thruster dynamics and saturation}

\section{Case VI: Compensation by RMD and RMA}

In the last simulation study, both thruster dynamics and saturation were considered, see Fig. 13. The RMD and the RMA methods were applied. The result were nearly similar to those of Case III.

\section{Conclusions}

It has been shown how reference model decomposition (RMD) can be applied to ROVs with thruster dynamics. A reference model adjustment (RMA) technique was used to compensate for the thruster saturation. Both methods were demonstrated on a simplified model of an ROV in yaw with promising results. Parameter drift was avoided in both cases. With RMA, the parameters converged to their true value. 


\section{ACKNOWLEDGMENTS}

This work was sponsored by The Royal Norwegian Council for Scientific and Industrial Research through the MOBATEL program at The Norwegian Institute of Technology. (The MOBATEL program is on model-based teleoperation of an underwater vehicle over a narrow-banded acoustic link.)

\section{REFERENCES}

AMERONGEN, J. VAN (1982). Adaptive steering of ships: a model-reference approach to improved manoeuvring and economical course keeping, Ph.D. thesis, Delft University of Technology, The Netherlands.

Butler, H., Honderd, G. and Amerongen, J. VAn (1991). Reference model decomposition in direct adaptive control, International Journal of Adaptive Control and Signal Processing, 5, 199-217.

Fossen, T. I. (1991). Nonlinear modelling and control of underwater vehicles, Dr. Ing. thesis, The Norwegian Institute of Technology, Trondheim, Norway.

Fossen, T. I. and SAGatun, S. I. (1991). Adaptive control of nonlinear underwater robotic systems, Proceedings of the IEEE Conference on Robotics and Automation, Sacramento, California, pp. 1687-1695.

HorowitZ, R. and TomizUKA, M. (1986). An adaptive control scheme for mechanical manipulators-compensation of nonlinearity and decoupling control, Technical Report no. 80-WA/DSC-6, ASME.

Popov, V. M. (1973). Hyperstability of Control Systems (Springer-Verlag, Berlin).

SLOTINE, J.-J. E. and LI, W. (1987). Adaptive manipulator control. A case study, Proceedings of the IEEE Conference on Robotics and Automation, Raleigh, North Carolina, pp. 1392-1400.

YoERGER, D. R., COOKE, J. G. and SLOTINE, J. -E. E. (1990). The influence of thruster dynamics on underwater vehicle behavior and their incorportation into control systems design, IEEE Journal of Oceanic Engineering, 15, 167-178.

YUH, J. (1990). Modeling and control of underwater vehicles, IEEE Transactions on Systems, Man and Cybernetics, 20, 1475. 